\title{
Remarkable simplicity in the prediction of nonspherical particle segregation
}

\author{
Ryan P. Jones $\odot,{ }^{1}$ Julio M. Ottino $\odot,{ }^{1,2,3}$ Paul B. Umbanhowar, ${ }^{1}$ and Richard M. Lueptow ${ }^{1,2,3, *}$ \\ ${ }^{1}$ Department of Mechanical Engineering, Northwestern University, Evanston, Illinois 60208, USA \\ ${ }^{2}$ Department of Chemical and Biological Engineering, Northwestern University, Evanston, Illinois 60208, USA \\ ${ }^{3}$ The Northwestern Institute on Complex Systems (NICO), Northwestern University, Evanston, Illinois 60208, USA
}

(Received 30 June 2020; accepted 13 October 2020; published 28 October 2020)

\begin{abstract}
Size-disperse mixtures of noncohesive particles segregate, or demix, during flow. For spherical particles, mixture segregation can be predicted based on the relative particle diameters. However, most particle systems in industry and geophysics involve nonspherical particles. Accounting for the immense range of particle shapes introduces additional parameters. As a proxy for nonspherical particles in general, we perform discrete element method simulations of gravity-driven free-surface flows of bidisperse mixtures of mm-sized particles that vary widely in their size and shape (disks, rods, and spheres). Remarkably, the propensity to segregate, measured in terms of a segregation length scale that characterizes the segregation velocity of the two species, can be predicted based on only the volume ratio of the two particle species. The segregation length scale increases linearly with the log of the volume ratio, as it does for bidisperse mixtures of spherical particles, independent of particle shape.
\end{abstract}

DOI: 10.1103/PhysRevResearch.2.042021

Introduction. Segregation, or demixing, in dense flows of noncohesive particles can occur due to particle size differences, where small particles percolate between large particles to lower positions in the flow, or particle density differences, where heavy particles sink while light particles rise in the flow, akin to buoyancy in fluids [1,2]. For spherical particles of equal density, it is trivial to predict which particles will rise or sink based on the particle diameter alone, even though there is still significant effort being made to understand the underlying physical mechanisms that drive the segregation process [3-5]. For nonspherical particles, the number of additional parameters describing particle shape substantially increases the complexity of the problem. The question is whether it is possible to capture the propensity for segregation in terms of a model as simple as Eq. (1) for spherical particles. This simplified practical model, based on "kinetic sieving" and "squeeze expulsion" of segregating spherical particles [6], approximates the segregation, or percolation (hence the $p$ subscript), velocity of species $i$ relative to the average flow for size-bidisperse mixtures of species $i$ and $j$, where $j$ denotes the other species, as

$$
w_{p, i}=S_{i} \dot{\gamma}\left(1-c_{i}\right),
$$

where $\dot{\gamma}=\partial u_{x} / \partial z$ is the local shear rate ( $u_{x}$ is the velocity in the bulk flow direction and $z$ is in the depthwise direction), and $c_{i}$ is the local concentration of species $i$. This model [Eq. (1)] was developed and is accurate for mixtures with

\footnotetext{
*r-lueptow@northwestern.edu

Published by the American Physical Society under the terms of the Creative Commons Attribution 4.0 International license. Further distribution of this work must maintain attribution to the author(s) and the published article's title, journal citation, and DOI.
}

similar concentrations of the two species $[7,8]$. For mixtures with widely varying species concentrations, the segregation velocity is more accurately described by a model that is second order in concentration $[9,10]$.

The segregation length scale for species $i, S_{i}$, is an empirical parameter that characterizes the propensity for a bidisperse mixture of spherical particles to segregate that depends on the ratio of diameters of the constituent species, $R_{D}=d_{i} / d_{j}$, and the diameter of the small species, $d_{s}=\min \left(d_{i}, d_{j}\right)$, as $[2,7,8]$

$$
S_{i} / d_{s}=0.26 \ln \left(R_{D}\right) .
$$

$w_{p, i}$ depends on the shear rate, since relative motion of particles is necessary for segregation to occur, and the concentration of the other particle species, $c_{j}=1-c_{i}$, since particles only segregate if they are in the presence of particles of the other species. The segregation direction, governed by $R_{D}$, is positive (upward) when the ratio is greater than 1 and vice versa.

While this approach works well for spherical particles, particles in both geophysical flows and many industrial processes are nonspherical. Particles can be rough and faceted in geophysics, mining, or agricultural processing, or geometric shapes, such as ellipsoids and pellets, in pharmaceutical or chemical processing. Given the bewilderingly large parameter space for particle shapes, predicting the segregation of mixtures of arbitrary nonspherical particles is quite challenging. Previous studies have considered segregation of nonspherical particles, including ellipsoids [11,12], cuboids and spheres [13], rods [14], and irregularly shaped particles [15], but the data are so limited that no universal scaling has been proposed.

Here, we consider a broad range of particle sizes and shapes using mm-sized cylindrical particles (rodlike and disklike) as well as spherical particles as a proxy for mixtures of arbitrary nonspherical particles. Accounting for a broad 

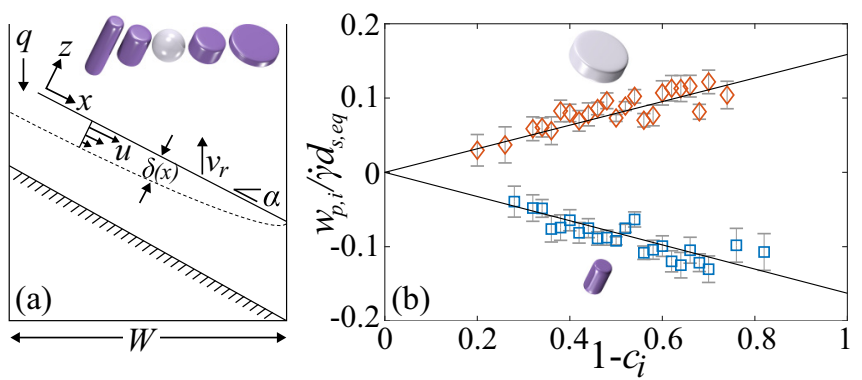

FIG. 1. (a) Schematic of 2D bounded heap with periodic boundary conditions in the $T$-wide $y$ direction. The coordinate system is rotated by the angle of repose $\alpha$ and rises at the heap-rise velocity $v_{r}$. The flowing layer shape is idealized and its thickness is exaggerated for purposes of illustration. Inset: Examples of equal-volume rodlike, spherical, and disklike particles with $0.185 \leqslant l / d \leqslant 4$. (b) Nondimensionalized segregation velocity dependence on concentration for $d=6.35 \mathrm{~mm}$ and $l=3.175 \mathrm{~mm}$ rods (blue $\circ$ ) and $d=3.175 \mathrm{~mm}$ and $l=4.75 \mathrm{~mm}$ disks (red $\diamond)$. Slope of the linear least-squares fits to Eq. (1) (lines) provides the segregation length-scale value $\left|S_{i} / d_{s, \text { eq }}\right|=0.17$.

range of particle shapes adds several additional parameters to the problem. Accordingly, we seek to determine if it is possible to represent the segregation of nonspherical particles in terms of an equation analogous to Eq. (1) and a parameter (or parameters) similar to $S_{i}$ having a functional dependence on the size and shape of the particles comprising the mixture analogous to Eq. (2).

Simulations. We conduct discrete element method (DEM) simulations [16-20] of superellipsoid particles in a gravitydriven free-surface flow [12-14,21-23]. The particle shape is determined by the inside-outside function for superellipsoids [24-26]

$$
P(\boldsymbol{X})=\left(\left|\frac{x}{a}\right|^{s}+\left|\frac{y}{b}\right|^{s}\right)^{t / s}+\left|\frac{z}{c}\right|^{t}-1,
$$

where $P(\boldsymbol{X})=0$ defines the particle surface. The radii $a$, $b$, and $c$ control the particle dimension along the $x, y$, and $z$ axes, respectively, and parameters $s$ and $t$ control corner sharpness as well as particle shape, which can vary from octahedral $(s=1, t=1)$ to cubic $(s \geqslant 8, t \geqslant 8)$. Here, we consider two particle shapes: cylinders $(s=2, t=8)$ and spheres $(s=2, t=2)$. To survey a broad range of shapes, we examine both rodlike and disklike cylindrical particles. Noting that cylinders have two length scales, the range of conditions for the bidisperse particle mixtures considered here can be expressed in terms of the diameter ratio, $1 / 3 \leqslant R_{D}=d_{i} / d_{j} \leqslant 1$, the length ratio, $1 \leqslant R_{L}=l_{i} / l_{j} \leqslant 4$, and a reference ratio, $1 / 3 \leqslant R_{*}=l_{j} / d_{i} \leqslant 3$, which together correspond to aspect ratios, $1 / 9 \leqslant l / d \leqslant 9$. We also consider mixtures of cylinders and spheres, varying the diameter ratio, $0.5 \leqslant R_{D} \leqslant 2$, and a modified length ratio, $0.5 \leqslant R_{L^{\prime}} \leqslant 2$, based on the cylinder length and the sphere diameter. Examples of particle shapes examined in this study are illustrated in Fig. 1(a) (inset), which shows equal-volume rodlike, disklike, and spherical particles.
Particle contact forces are based on calculating the greatest overlap of the contacting particle pair, which is found by Lagrangian optimization $[27,28]$ combined with screening algorithms of spatial hashing, bounding sphere contact evaluation, and object oriented bounding box contact detection $[14,22]$ to reduce the number of times the contact algorithm is applied to noncontacting particles. Particle stiffness and damping are based on the restitution coefficient, 0.8 , and binary collision time, $10^{-3} \mathrm{~s}[7,19]$. Particle-particle contacts use a friction coefficient of 0.4. A simulation time step of $2.5 \times 10^{-5} \mathrm{~s}$ provides sufficient resolution of particle deformation during contact [20]. Simulations are performed using an in-house DEM code that has been validated against experiments for spherical particles (see Ref. [2] and references therein), and mixtures of disk- and rodlike particles [29].

Simulations are performed for flow on a single-sided, two-dimensional (2D) bounded heap, shown schematically in Fig. 1(a), by feeding particles vertically on one side of a spanwise periodic box with spanwise extent $T / l_{\max }=8$ and width $W / l_{\max }=200$, where $l_{\max }$ is the diameter of the minimum bounding sphere of the larger particle species in each simulation. Steady state segregation occurs in a thin flowing layer of thickness $\delta(x)$ as particles flow down the surface of the heap toward the wall opposite the feed zone. The angled bottom bounding wall reduces the total number of particles needed in the simulation. Particles fall onto the heap at a $2 \mathrm{D}$ feed rate, $17 \leqslant q=Q / T \leqslant 130 \mathrm{~cm}^{2}$, where $Q$ is the volumetric feed rate. For each simulation, the volumetric flow rate varies from the feed rate at the top of the heap to 0 at the downstream end wall. This variation in flow rates creates a wide range of shear rates of $0.01 \leqslant \dot{\gamma} \leqslant 726 \mathrm{~s}^{-1}$ across all simulations. The coordinate system rises with the surface at a steady rise velocity, $v_{r}=q / W$, so in its frame of reference the flow and segregation are steady. To quantify the segregation, particle data are spatially and temporally averaged in quadrilateral bins oriented parallel to and rising with the free surface $\left(l_{\max } / 2\right.$ in the depthwise, $2 l_{\max }$ in the streamwise direction, and $T$ in the spanwise direction) to find the bulk and species velocity, $\boldsymbol{u}$ and $\boldsymbol{u}_{i}$, respectively, the segregation velocity, $w_{p, i}=w-w_{i}$, and species concentration $c_{i}$ throughout the flowing layer, as in previous studies [8,10,30,31]. Particles overlapping multiple bins have their weighted partial volume applied to each overlapped bin.

Segregation of cylindrical particles. Figure 1(b) shows an example of the segregation velocity $w_{p, i}$ scaled by $\dot{\gamma}$ and a particle-based length scale that will be explained shortly, $d_{s, \text { eq }}$, as in previous studies $[8,10]$, versus concentration for a bidisperse mixture of rods and disks. The data are sampled throughout the entire flowing layer, resulting in a wide range of values for $w_{p, i}, \dot{\gamma}$, and $c_{i}$. The data fall along two lines, one for the rising species (disk) and one for the sinking species (rod), that both intersect the origin. This result is consistent with Eq. (1) for spherical particles, where the segregation length scale $S_{i}$ is the slope of the least-squares linear fit of the data. Similar results to those shown in Fig. 1(b) occur for all other particle mixtures considered here, thereby demonstrating the validity of Eq. (1) for nonspherical particles in addition to spherical particles for which it was originally 


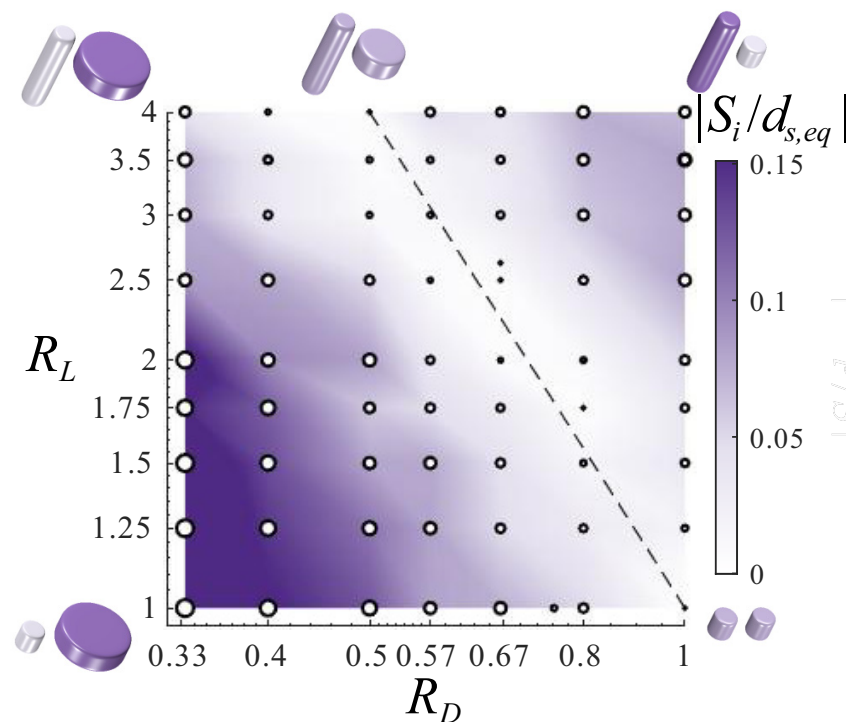

FIG. 2. Absolute value of the nondimensionalized segregation length scale of the longer species $i,\left|S_{i} / d_{s, \text { eq }}\right|$, vs length ratio $R_{L}$ and diameter ratio $R_{D}$ for $R_{*}=1$. Data symbol (o) diameter is proportional to $\left|S_{i} / d_{s, \mathrm{eq}}\right|$ and color contours are interpolated from data points. The equal volume ratio dashed line, $R_{V}=1$, aligns with the region where $S_{i} / d_{s, \text { eq }}$ is near zero. Representative particles of both species, with purple representing the rising species, are shown at each corner and the top of the equal-volume line.

validated. The linear dependence of $w_{p, i}$ for a range of $c_{i}$ confirms similar results for a more limited study of cylindrical particles [14].

The challenge now is to determine an appropriate length scale to normalize $S_{i}$ and then to determine the dependence of $S_{i}$ on particle shape and size. Previous studies for mixtures of spheres have shown that $S_{i}$, which has units of length, when normalized by the small particle diameter $d_{s}$, can be represented solely as a function of a relevant ratio of a particle property, such as the diameter or density ratio [8,10,31] [for example, Eq. (2)]. Although the appropriate length scale to nondimensionalize $S_{i}$ is not obvious for nonspherical particles, it should be on the order of the particle size as it is for spherical particles (as opposed to a length scale based on the dimensions of the experimental apparatus). Here, we use the diameter of the volume equivalent sphere of the smaller volume species $d_{s \text {, eq }}$ as the characteristic particle length scale. Using other characteristic particle length scales (e.g., radius of gyration) does not improve, and in some cases makes much worse, the collapse of the data for the results discussed below.

To determine the dependence of the propensity for segregation, characterized by $S_{i} / d_{s \text {, eq }}$, on particle shape and size, we consider a broad range of bidisperse cylindrical particle mixtures. Figure 2 shows the dependence of $\left|S_{i} / d_{s \text {, eq }}\right|$ for the longer species $(i)$ on the length ratio $R_{L}$ and the diameter ratio $R_{D}$ for 65 simulations with reference ratio $R_{*}=l_{j} / d_{i}=1$, which corresponds to the aspect ratio for the monodisperse mixture (lower right-hand corner of the figure). The longer species rises in the upper right-hand portion of the figure and sinks in the lower left-hand side of the figure. The segregation length scale of the shorter species $j, S_{j} / d_{s \text {, eq }}$, which is not shown, is of opposite sign and of nearly equal magnitude to $S_{i} / d_{s, \text { eq }}$, having an rms deviation from $-S_{i} / d_{s \text {, eq }}$ of $7 \%$ across all 65 measurements.

Figure 2 demonstrates that longer rods rise in the presence of shorter rods (upper right), and larger diameter disks rise in the presence of smaller diameter disks (lower left). The segregation is strongest when the volume of the two particles differs substantially (upper right and lower left). A region exists between these two extremes where the particles remain mixed $\left(S_{i} \approx S_{j} \approx 0\right.$ ) that corresponds to a volume ratio, $R_{V}=$ $V_{i} / V_{j}=1$, shown as a dashed line in Fig. 2.

The dependence of segregation on the relative volumes of the two particle species is further explored by changing the reference ratio, $1 / 3 \leqslant R_{*} \leqslant 3$, with $1<R_{L}<4$ and $1 / 3<$ $R_{D}<1 . R_{*}=1 / 3$ corresponds to mixtures of disklike particles, and $R_{*}=3$ corresponds to mixtures of rodlike particles. Although these data are omitted for brevity, in all cases $w_{p, i}$ follows the form of Eq. (1), and mixtures with particles having a larger difference in volume segregate more, while particles of approximately equal volume do not segregate.

Much as the segregation of rodlike and disklike particles, DEM simulations for mixtures of spheres and cylinders (rods or disks) demonstrate that the particle species with the greater volume rises, and the particle species with the smaller volume sinks. And again, when the particles have equal volumes, there is negligible segregation.

The observation that higher volume particles always segregate upward while smaller volume particles sink downward, and the related observation that particles of equal volume remain mixed strongly suggest that the segregation length scale $S_{i} / d_{s \text {,eq }}$ depends on the volume ratio $R_{V}$. To test this hypothesis, Fig. 3 plots $S_{i} / d_{s \text {, eq }}$ vs $R_{V}$ for all of the

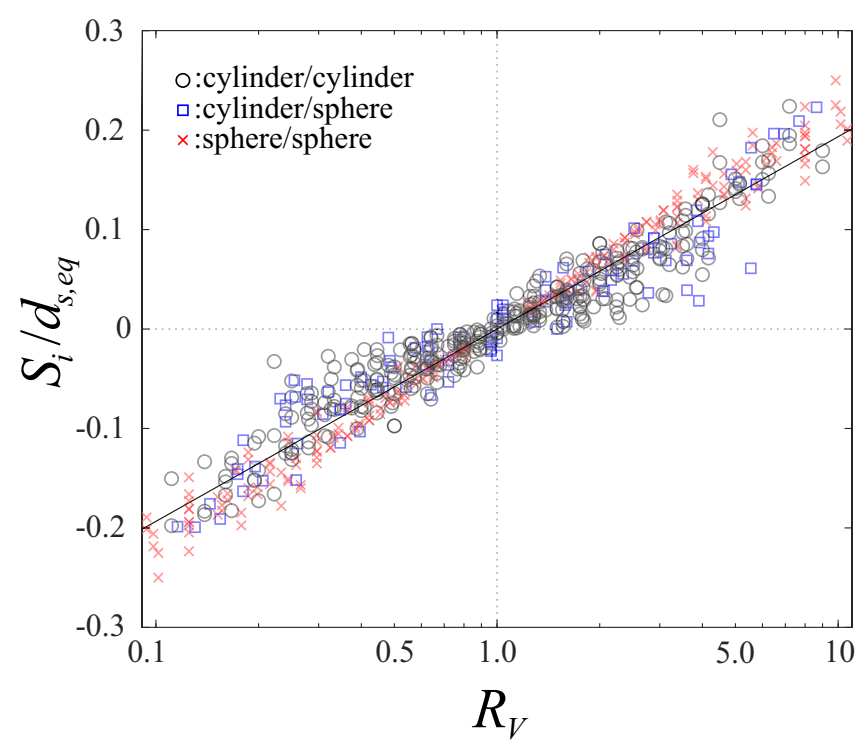

FIG. 3. Nondimensional segregation length scale $S_{i} / d_{s \text {, eq }}$ for the longer species vs volume ratio $R_{V}$ for bidisperse mixtures of cylinders (black $\circ$ ), cylinders and spheres (blue $\square$ ), and spheres (red $\times$ ) [8]. Symbols are semitransparent so overlapping points are more intense in color. The linear least-squares fit to the data (line) for all mixtures to $S_{i} / d_{s, \text { eq }}=C \ln \left(R_{V}\right)$ has a slope $C=0.082$. 
parameter combinations considered: 325 simulations of bidisperse mixtures of cylinders $\left(1 \leqslant R_{L} \leqslant 4,1 / 3 \leqslant R_{D} \leqslant 1\right.$, and $1 / 3 \leqslant R_{*} \leqslant 3$, corresponding to $1 / 9 \leqslant l / d \leqslant 9$ ) and 54 simulations of bidisperse mixtures of spheres and cylinders $\left(1 / 2 \leqslant R_{L^{\prime}} \leqslant 2\right.$ and $\left.1 / 2 \leqslant R_{D} \leqslant 2\right)$. In addition, data for 146 simulations of size-bidisperse mixtures of spherical particles $\left(1 / 2.2 \leqslant R_{D} \leqslant 2.2\right)$ from previous work [8] are also included in the figure. There are two data points for each simulation: one for one species at a particular value of $R_{V}$ and another for the other species at $1 / R_{V}$.

Figure 3 demonstrates that particle segregation characterized by $S_{i} / d_{s \text {, eq }}$ is, to first order (coefficient of determination, $\left.R^{2}=0.894\right)$, linear with $\ln \left(R_{V}\right)$,

$$
S_{i} / d_{s, \mathrm{eq}}=C \ln \left(R_{V}\right)
$$

where $C=0.082$, a form that is surprisingly consistent with Eq. (2) for spherical particles. Again, the segregation direction is governed by $R_{V}$ to be positive (upward) when the ratio is greater than 1 and vice versa. While there is some scatter in the data, it is remarkable that the segregation of particles having such widely varying shapes and sizes, a range broader than even the extremes shown in Fig. 1(a) (inset), can be condensed to this simple correlation. In addition to the volume ratio $R_{V}$, we considered several other ways to characterize the two particle species including surface area, projected surface area in the direction of segregation, and sphericity, but none collapse the data better than $R_{V}$.

While the dependence of segregation on the volume ratio is clear, the physics associated with this result is not. Nevertheless, the volume-based dependence for the segregation of nonspherical particles found here may open up different perspectives. In previous studies of a single spherical intruder in a bed of sheared spherical particles [3-5], the direction of segregation is consistent with the results presented here. However, at much larger volume ratios than those studied here (greater than about 64), a larger intruder sinks due to its increased weight relative to the upward contact forces from the smaller bed particles [5]. Further demonstrating the complexity of the physics associated with segregation, in bidisperse mixtures of spherical particles that vary in both volume and density, the segregation depends not only on the volume ratio and density ratio [32] but also on the species concentration [33-35]. Therefore, the physics of segregation is, at least in part, dependent on both the relative size, here characterized by the volume ratio, and the mass (or density) ratio between particle species. This complex interaction is not fully understood even in the dilute limit of a single intruder in spherical particle systems, although it appears to be related to both particle contact anisotropy and flow kinematics [5]. The physics is likely even more challenging for the more complicated particle contact interactions and kinematics associated with nonspherical particles, but the volume-based segregation dependence found here is in accord with recent results suggesting a segregation force based on a size-corrected buoyancy [5].

Conclusions. In the end, characterizing the propensity for segregation is quite simple, even for particle shapes and sizes varying even more widely than those shown in Fig. 1(a) (inset). First, just as with spherical particles, the segregation velocity is to first order a function of only the shear rate and the concentration of the particle species [Eq. (1)]. Second, the segregation length scale $S_{i}$ that characterizes the propensity for segregation depends only on the volume ratio of the two particles via the simple relation $S_{i} / d_{s \text {, eq }}=C \ln \left(R_{V}\right)$, where $C=0.082$ is constant across a wide range of cylindrical and spherical particle mixtures. A more detailed presentation of the simulations and conclusions, including data omitted for brevity above, will be presented in an upcoming publication.

The similarity in segregation for mixtures of particle species as different as those in Fig. 1(a) (inset) suggests that predicting segregation between differently shaped particles may be as simple as determining $R_{V}$, at least to first order. That is, generally spheroidal particles, including particles with faceted surfaces, ellipsoids, tablet- or capsule-shaped particles, and seed-or grain-shaped particles, would likely follow the same volume-ratio-based dependence for segregation, given that these particle shapes rarely differ from one another as much as the extreme examples of disks and rods differ in Fig. 1(a) (inset). Further study is needed to confirm this prediction, but the simple relationship $S_{i} / d_{s, \mathrm{eq}}=C \ln \left(R_{V}\right)$ is of great benefit in modeling segregation. Of course, this relationship is unlikely to hold for extreme particle shapes, e.g., concave shapes that interact through interlocking behavior or large aspect ratio rods (fibers) that strongly align with the flow or locally jam. ${ }^{1}$ Likewise, particles differing appreciably in density would not follow this relation. Nevertheless, Fig. 3 demonstrates that the segregation of a large and important subset of all nonspherical particle mixtures, namely cylinder-cylinder mixtures, cylinder-sphere mixtures, and sphere-sphere mixtures, can be simply predicted based on the relative volumes of the particles, regardless of particle shape.

Acknowledgments. This work was funded by The Dow Chemical Company.

\footnotetext{
${ }^{1}$ Note that the model may also be somewhat less accurate when particle orientation is evolving, such as the upstream flowing layer in rotating tumblers or near the feed zone in heaps. However, once the particle orientation is established, the model should apply to a wide range of gravity-driven free-surface flows just as it does for spherical particles [2].
}

[1] J. M. N. T. Gray, Annu. Rev. Fluid Mech 50, 407 (2018).

[2] P. B. Umbanhowar, R. M. Lueptow, and J. M. Ottino, Annu. Rev. Chem. Biomol. Eng. 10, 129 (2019).

[3] F. Guillard, Y. Forterre, and O. Pouliquen, J. Fluid Mech. 807, R1 (2016).
[4] K. van der Vaart, M. P. van Schrojenstein Lantman, T. Weinhart, S. Luding, C. Ancey, and A. R. Thornton, Phys. Rev. Fluids 3, 074303 (2018).

[5] L. Jing, J. M. Ottino, R. M. Lueptow, and P. B. Umbanhowar, Phys. Rev. Res. 2, 022069(R) (2020). 
[6] S. B. Savage and C. K. K. Lun, J. Fluid Mech. 189, 311 (1988).

[7] Y. Fan, C. P. Schlick, P. B. Umbanhowar, J. M. Ottino, and R. M. Lueptow, J. Fluid Mech. 741, 252 (2014).

[8] C. P. Schlick, Y. Fan, A. B. Isner, P. B. Umbanhowar, J. M. Ottino, and R. M. Lueptow, AIChE J. 61, 1524 (2015).

[9] P. Gajjar and J. M. N. T. Gray, J. Fluid Mech. 757, 297 (2014).

[10] R. P. Jones, A. B. Isner, H. Xiao, J. M. Ottino, P. B. Umbanhowar, and R. M. Lueptow, Phys. Rev. Fluids 3, 094304 (2018).

[11] H.-G. Matuttis, N. Ito, and H. Watanabe, in Proceedings of Traffic and Granular Flow '01 (Springer, Berlin, 2003), pp. 455-460.

[12] S. Y. He, J. Q. Gan, D. Pinson, and Z. Y. Zhou, Powder Technol. 341, 157 (2019).

[13] G. G. Pereira and P. W. Cleary, Granular Matter 19, 23 (2017).

[14] Y. Zhao, H. Xiao, P. B. Umbanhowar, and R. M. Lueptow, AIChE J. 64, 1550 (2018).

[15] M. Alizadeh, A. Hassanpour, M. Pasha, M. Ghadiri, and A. Bayly, Powder Technol. 319, 313 (2017).

[16] P. A. Cundall and O. D. L. Strack, Geotechnique 29, 47 (1979).

[17] Y. Guo and J. S. Curtis, Annu. Rev. Fluid Mech 47, 21 (2015).

[18] J. Schäfer, S. Dippel, and D. E. Wolf, J. Phys. I (France) 6, 5 (1996).

[19] L. E. Silbert, G. S. Grest, R. Brewster, and A. J. Levine, Phys. Rev. Lett. 99, 068002 (2007).
[20] G. H. Ristow, Pattern Formation in Granular Materials, Springer Tracts in Modern Physics Vol. 164 (Springer, Berlin, 2000).

[21] Y. You and Y. Zhao, Powder Technol. 331, 179 (2018).

[22] G. Lu, J. R. Third, and C. R. Müller, Chem. Eng. Sci. 78, 226 (2012).

[23] P. W. Cleary, Powder Technol. 248, 103 (2013).

[24] A. H. Barr, IEEE Comput. Graph. Appl. 1, 11 (1981).

[25] A. Jaklič and F. Solina, IEEE Trans. Syst., Man, Cybern. B. Cybern. 33, 648 (2003).

[26] S. Zhao, N. Zhang, X. Zhou, and L. Zhang, Powder Technol. 310, 175 (2017).

[27] G. T. Houlsby, Comput. Geotech. 36, 953 (2009).

[28] X. Lin and T.-T. Ng, Int. J. Numer. Anal. Methods Geomech. 19, 653 (1995).

[29] R. P. Jones, Modeling segregation in spherical and nonspherical granular materials, Ph.D. thesis, Northwestern University, 2020.

[30] C. P. Schlick, Y. Fan, P. B. Umbanhowar, J. M. Ottino, and R. M. Lueptow, J. Fluid Mech. 765, 632 (2015).

[31] H. Xiao, P. B. Umbanhowar, J. M. Ottino, and R. M. Lueptow, Proc. R. Soc. A 47220150856 (2016).

[32] D. R. Tunuguntla, O. Bokhove, and A. R. Thornton, J. Fluid Mech. 749, 99 (2014).

[33] D. R. Tunuguntla and A. R. Thornton, EPJ Web Conf. 140, 03079 (2017).

[34] N. Thomas, Phys. Rev. E 62, 961 (2000).

[35] A. Tripathi and D. V. Khakhar, J. Fluid Mech. 717, 643 (2013). 